\title{
A RESULT ON HERMITIAN OPERATORS
}

\section{by J. E. JAMISON and PEI-KEE LIN $\dagger$}

(Received 15 September, 1987)

1. Introduction. Let $X$ be a complex Banach space. For any bounded linear operator $T$ on $X$, the (spatial) numerical range of $T$ is defined as the set

$$
V(T)=\left\{f(T x): x \in X, f \in X^{*},\|x\|=\|f\|=1=f(x)\right\} .
$$

If $V(T) \subseteq \mathbf{R}$, then $T$ is called hermitian. Vidav and Palmer (see Theorem 6 of [3, p. 78] proved that if the set $\{H+i K: H$ and $K$ are hermitian $\}$ contains all operators, then $X$ is a Hilbert space. It is natural to ask the following question.

Question. Is $X$ a Hilbert space if $\{H+i K: H$ and $K$ are hermitian $\}$ contains all compact operators?

In this article, we have proved the following theorem.

THEOREM. Let $P$ be a norm-1 projection on $X$. If there exist two hermitian operators $H$ and $K$ such that $P=H+i K$, then $P$ is hermitian and $P=H$.

Recall that an element $x \in X$ is hermitian if the span of $x$ is the range of a rank-1 hermitian projection $P \in \mathscr{L}(X)$. Berkson [1] (also see [5, p. 499] proved that if every nonzero element is hermitian, then $X$ is isometrically isomorphic to a Hilbert space. Hence, the theorem shows the answer of the above question is affirmative.

2. Proof of theorem. Let $Y=\operatorname{range} P$ and $Z=\operatorname{ker} P$. Then the matrices of $H, K: X=Y \oplus Z \rightarrow X=Y \oplus Z$ have the following forms:

$$
H=\left(\begin{array}{cc}
E+I_{Y} & F \\
C & D
\end{array}\right)
$$

and

$$
K=\left(\begin{array}{ll}
i E & i F \\
i C & i D
\end{array}\right)
$$

where $E: Y \rightarrow Y, I_{Y}$ is the identity on $Y, F: Z \rightarrow Y, C: Y \rightarrow Z$ and $D: Z \rightarrow Z$. Since $H$ and $K$ are hermitian it follows that

$$
i[H, K]=i H K-i K H=H(P-H)-(P-H) H=H P-P H=\left(\begin{array}{cc}
0 & -F \\
C & 0
\end{array}\right)
$$

† Research of this author partially supported by NSF. The authors wish to thank Tom Barton for valuable discussions concerning these results. They would also like to thank the referee for his corrections to a previous version.

Glasgow Math. J. 31 (1989) 71-72. 
is hermitian (see Lemma 5.4 of [3]). If $[H, K]=0$ (i.e. $P$ is normal), then $Y$ and $Z$ are invariant subspaces of $H$ and $K$. So the restrictions of $H$ and $K$ to $Y$ and $Z$ are hermitian. This implies that

$$
I_{Y}=\left.H\right|_{Y}+\left.i K\right|_{Y} \text { and } 0=\left.H\right|_{Z}+\left.i K\right|_{Z}
$$

So by Lemma 1.1 of [6], $I_{Y}=\left.H\right|_{Y},\left.K\right|_{Y}=0$ and $\left.H\right|_{Z}=0=\left.K\right|_{Z}$. Now, we claim that $[H, K]=0$. It is known [6] that if $T$ is a non-zero hermitian operator on $X$, the ultraproduct $\tilde{T}$ of $T$ has at least one non-zero eigenvalue (for definition and detail, see [6]). Moreover, if $T$ is hermitian and $T(Y) \subseteq Z$, then $\tilde{T}$ is hermitian and $\tilde{T}(\tilde{Y}) \subseteq \tilde{Z}$, where $\tilde{Z}=\left\{\left(z_{i}\right) \in \bar{X}: z_{i} \in X\right\}$ and $\bar{Y}=\left\{\left(y_{i}\right) \in \bar{X}: y_{i} \in Y\right\}$. So we may assume $i[H, K]$ has a non-zero real eigenvalue $\lambda$. Let $x=y \oplus z$ be a corresponding eigenvector. Then $i[H, K] y=\lambda z$ and $i[H, K] z=\lambda y$ (since $i[H, K] Y \subseteq Z$ and $i[H, K] Z \subseteq Y$ ). So $y \neq 0 \neq z$ (we can assume that $\|y\|=1$ ), and there exist $y^{\prime} \in Y$ and $z^{\prime} \in Z$ such that

$$
H y=y^{\prime}+\lambda z \text { and } H z=-\lambda y+z^{\prime} \text {. }
$$

Therefore,

$$
\begin{aligned}
{[i[H, K], H] y } & =i[H, K] H y-i H[H, K] y \\
& =i[H, K]\left(y^{\prime}+\lambda z\right)-\lambda H z \\
& =i[H, K] y^{\prime}+\lambda^{2} y+\lambda^{2} y-\lambda z^{\prime} .
\end{aligned}
$$

Since $P$ is a norm-1 projection onto $Y$, there is $f \in X^{*}$ such that $\|f\|=1=\|y\|=f(y)$ and $\left.f\right|_{z}=0$. So

$$
\left.f([i[H, K], H] y)=2 \lambda^{2} \quad \text { (since } i[H, K] y^{\prime} \in Z\right) .
$$

This contradicts the fact that $i[i[H, K], H]$ is hermitian.

\section{REFERENCES}

1. E. Berkson, Some types of Banach spaces, hermitian operators, and Bade functionals, Trans. Amer. Math. Soc. 116 (1965), 376-385.

2. E. Berkson, A characterization of complex Hilbert spaces, Bull. London Math Soc. 2 (1970), 313-315.

3. F. F. Bonsall and J. Duncan, Numerical ranges of operators on normed spaces and of elements of normed algebras, London Math. Soc. Lecture Notes series, 2 (C.U.P., 1971).

4. F. F. Bonsall and J. Duncan, Numerical ranges II, London Math. Soc. Lecture Notes Series, 10 (C.U.P., 1973).

5. N. J. Kalton and G. V. Wood, Orthonormal systems in Banach spaces and their applications, Math. Proc. Camb. Phil. Soc. 76 (1976), 493-510.

6. K. Mattila, Normal operators and proper boundary points of the spectra of operators on a Banach space, Ann. Acad. Sci. Fenn. Ser, AI, Math. Dissertations 19 (1978).

Department of Mathematics

Memphis STATE UNIVERSITY

MEMPHIS

TENNESSEE 38152, U.S.A. 